\title{
Prostate cancer grading: a decade after the 2005 modified system
}

\author{
Jonathan I Epstein ${ }^{1,2,3}$ \\ ${ }^{1}$ Department of Pathology, The Johns Hopkins Medical Institution, Baltimore, MD, USA; ${ }^{2}$ Department \\ Urology, The Johns Hopkins Medical Institution, Baltimore, MD, USA and ${ }^{3}$ Department of Oncology, The \\ Johns Hopkins Medical Institution, Baltimore, MD, USA
}

\begin{abstract}
This review article will cover the evolution of grading of prostate cancer from the original Gleason system in the 1960-1970s to a more patient-centric grading system proposed in 2013 from a group at Johns Hopkins Hospital, validated in 2014 by a large multi-institutional study, and subsequently accepted by the World Health Organization (WHO), College of American Pathology (CAP), and the AJCC TNM system. Covered topics include: (1) historical background; (2) 2005 and 2014 International Society of Urological Pathology Grading Conferences; (3) Description of Gleason patterns; (4) new approaches to display Gleason grades; (5) grading variants and variations of acinar adenocarcinoma; (6) reporting rules for Gleason grading reporting secondary patterns of higher grade when present to a limited extent; (7) reporting secondary patterns of lower grade when present to a limited extent; (8) reporting percentage pattern 4; (9) general applications of the Gleason grading system; (10) needle biopsy with different cores showing different grades; (11) radical prostatectomy specimens with separate tumor nodules; and (12) a new grading system for prostate cancer.

Modern Pathology (2018) 31, S47-S63; doi:10.1038/modpathol.2017.133
\end{abstract}

\section{Historical background}

Donald F Gleason in 1966 created a unique grading system for prostatic carcinoma based solely on the architectural pattern of the tumor. ${ }^{1,2}$ Another innovative aspect of this system was, rather than assigning the worst grade as the grade of the carcinoma, the grade was defined as the sum of the two most common grade patterns and reported as the Gleason score. The original description of this system was based on a study of 270 patients from the Minneapolis Veterans Administration Hospital.

Initially, Gleason intended to classify carcinomas into four patterns, but a small group of distinctive tumors (clear cell) was observed and they were placed in a separate fifth category (pattern 4). Certain aspects of the original Gleason system would be interpreted differently in today's practice. The cribriform pattern described as a component of Gleason's original patterns 2 and 3 would today typically be considered higher grade. Individual cells listed under Gleason's original pattern 3 would

Correspondence: Dr JI Epstein, MD, Department of Pathology, The Johns Hopkins Medical Institution, 401N Broadway Street, Weinberg Building, Room 2242, Baltimore, MD 21231, USA.

E-mail: jepstein@jhmi.edu

Received 14 June 2017; revised 31 July 2017; accepted 1 August 2017 also be currently assigned a higher grade. Pattern 4 has become significantly expanded beyond Gleason's original description of tumors with clear cytoplasm that resembled renal cell carcinoma.

By 1974, Gleason and the Veterans Administration Cooperative Urological Research Group expanded their study to 1032 men. Gleason pattern 4 was described in a figure legend as 'raggedly infiltrating, fused-glandular tumor, frequently with pale cells, may resemble hypernephroma of kidney.' The Gleason system was further refined by Mellinger in 1977 when the papillary and cribriform tumor under Gleason pattern 3 was described as having a 'smooth and usually rounded edge.' In describing the breakdown of Gleason patterns among 2911 cases, Gleason pattern 1 was seen in $3.5 \%$; pattern 2 in $24.4 \%$; pattern 3 in $87.7 \%$; pattern 4 in $12.1 \%$; and pattern 5 in $22.6 \%$. These percentages added up to $\sim 150 \%$, as $50 \%$ of the tumors showed at least two different patterns.

In 1977, Gleason provided additional comments concerning the application of the Gleason system. ${ }^{3}$ 'Grading is performed under low magnification (x40100).' He also stated that 'an occasional small area of fused glands did not change a pattern 3 tumor to pattern 4. A small focus of disorganized cells did not change a pattern 3 or 4 tumor to pattern 5.' The only comment relating to tertiary patterns was 'occasionally, small areas of a third pattern were observed.' An 
iconic schematic diagram was drawn by Gleason that achieved widespread popularity (Figure 1).

In 2000, an influential editorial was written that Gleason scores 2-4 should not be assigned to cancer on needle biopsy for several reasons: (1) poor reproducibility even among experts; (2) poor correlation with prostatectomy grade with almost all cases showing higher grade at resection; and (3) a diagnosis of Gleason scores 3 and 4 may misguide clinicians and patients into believing that the patient has an indolent tumor. ${ }^{4}$ In a prior study from Johns Hopkins, we reported that only $1.6 \%$ of biopsies from other institutions were graded as Gleason scores $2-4$ in $2002-2003$, compared with $22.3 \%$ in 1994. ${ }^{5}$ Consequently, most of the lesions that appear to be very low grade on needle biopsies are currently diagnosed by urological pathologists as Gleason score $3+3=6$.

\section{5 and 2014 International Society of Urological Pathology grading conferences}

A group of urological pathologists convened at the 2005 United States and Canadian Academy meeting in San Antonio in an attempt to achieve consensus in controversial areas relating to the Gleason grading system. ${ }^{6}$ The aim of the meeting was to achieve consensus among leading urological pathologists in specific areas of Gleason grading, including areas where there was either a lack of data or scant information as to the optimal method of grading. Over 70 urological pathologists from around the world were invited to attend, with most attending. In 2014, over 80 expert pathologists, urologists, medical oncologists, and radiation oncologists attended a meeting in Chicago to: (1) resolve issues that lacked consensus in the earlier meeting; (2) deal with issues that were not discussed in the 2005 meeting; and (3) update guidelines based on interim research. ${ }^{7}$

\section{Gleason patterns}

\section{Gleason Score 1+1 = 2}

Gleason score of $1+1=2$ is a grade that should not be diagnosed regardless of the type of specimen, with extremely rare exception. Most cases that were diagnosed as Gleason score $1+1=2$ in the era of Gleason would today be referred to as adenosis (atypical adenomatous hyperplasia).

\section{Gleason Scores 3 and 4}

These low-grade tumor scores are occasionally assigned on transurethral resection specimens (TURPs) and in multifocal low-grade tumors within radical prostatectomy (RP) specimens. It is now accepted that Gleason scores 2-4 should not be

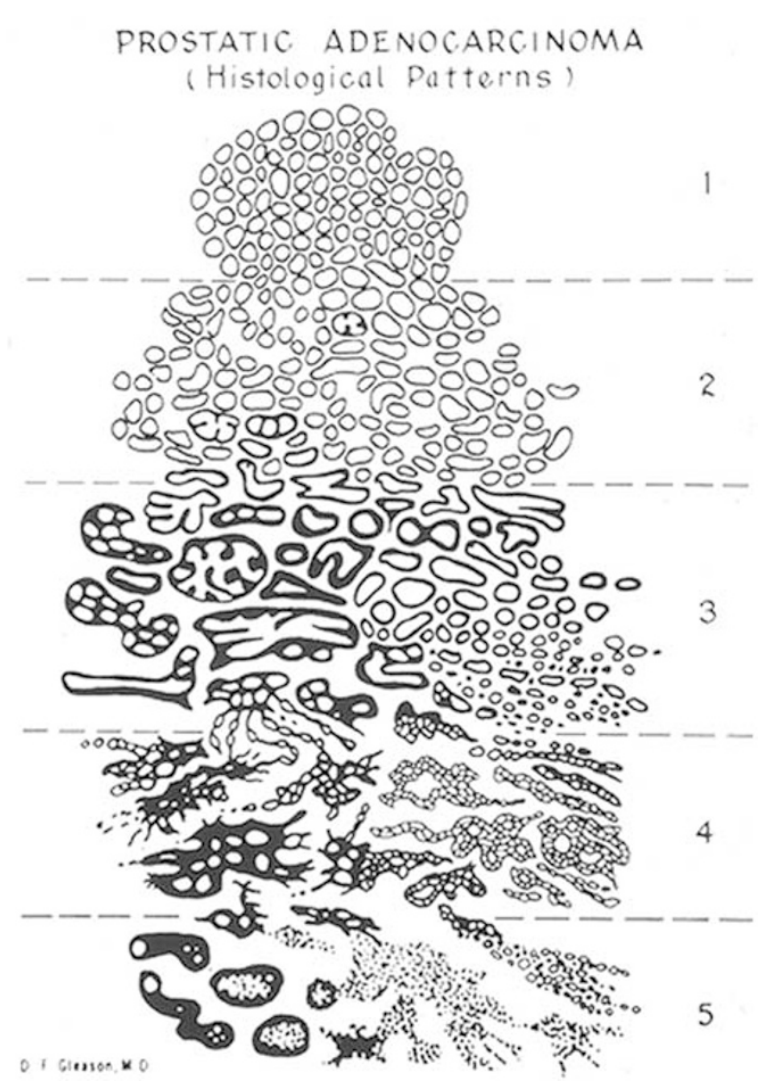

Figure 1 Original Gleason schematic grading diagram.

assigned to cancer on needle biopsy for several reasons as described above.

\section{Gleason Pattern 3}

A departure from the original Gleason classification system is that 'individual cells' would not be allowed within Gleason pattern 3. Rather, Gleason pattern 3 cancer consists of variably sized individual glands. A further area of divergence from the original Gleason system is the controversial area of cribriform Gleason pattern 3. Within Gleason's original illustrations of his cribriform pattern 3, he depicts large, cribriform glands that the consensus panel would uniformly diagnose as cribriform pattern 4. Before 2005, it was the routine to grade large cribriform glands as pattern 3 (Figure 2). ${ }^{8,9}$ In 2005, the consensus required extremely stringent criteria for the diagnosis of cribriform pattern 3 , with remaining cribriform patterns typically falling into Gleason pattern 4. The 2005 criteria used to diagnose cribriform pattern 3 were rounded, well-circumscribed glands of the same size of normal glands with uniform round evenly distributed lumina. When various images were shown to the consensus panel of potential candidates for cribriform Gleason pattern 3, almost none of them met the criteria based on subtle features, such as slight irregularities of the outer border of the cribriform glands. Subsequent to the 2005 meeting, this author reviewed 3590 

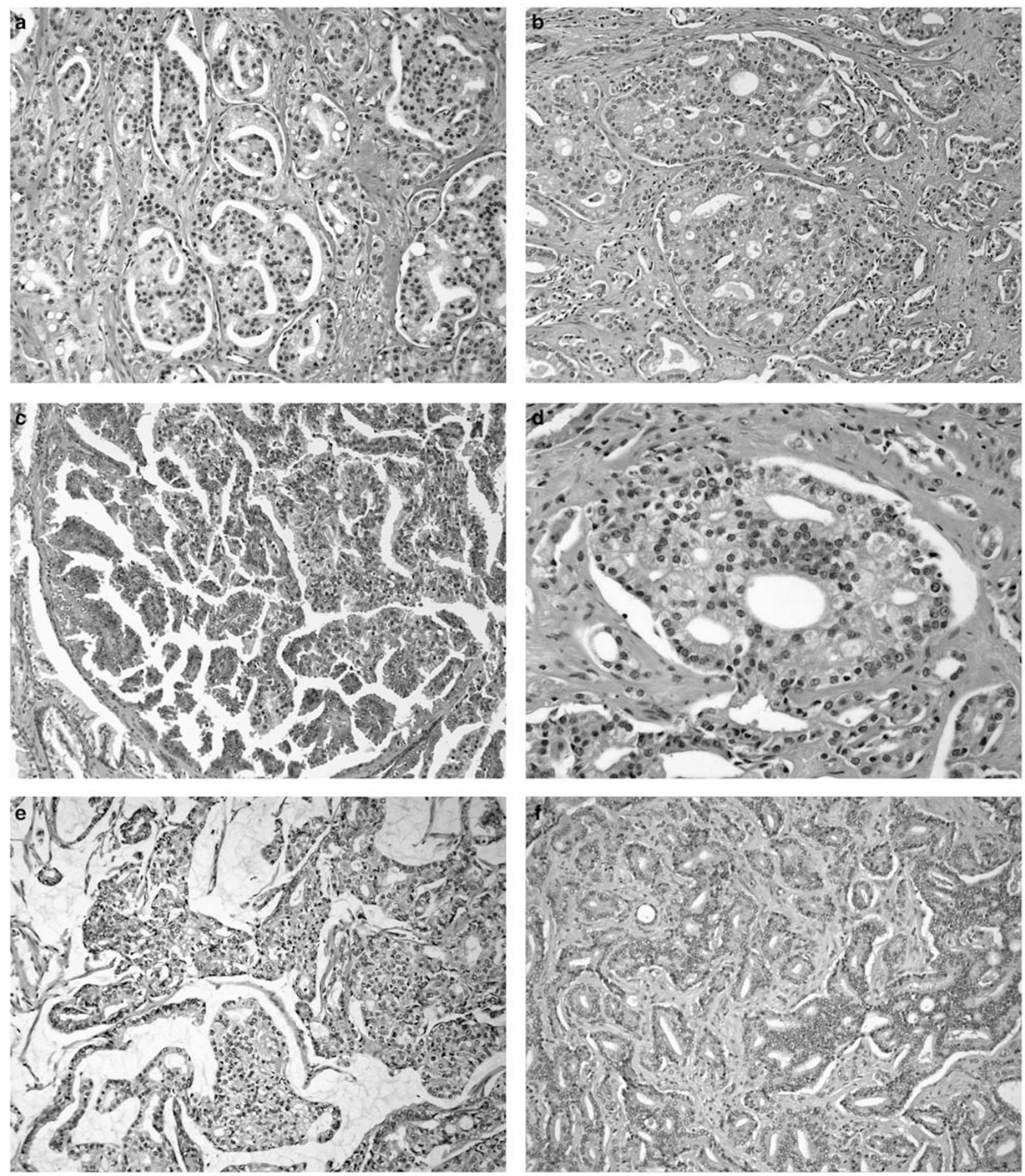

Figure 2 Images graded from several large academic prostate centers before 2005 as Gleason score $3+3=6$ with lymph node metastases. Currently, all these images would be graded as having a component of Gleason pattern 4. (a) Glomeruloid glands transitioning to small cribriform glands. Original magnification, 20x. (b) Medium to large irregular cribriform glands. Original magnification, 20x. (c) Papillary prostatic ductal adenocarcinoma. Original magnification, 20x. (d) Small more regular cribriform gland. Original magnification, 40x. (e) Cribriform glands floating within mucin. Original magnification, 10x. (f) Medium-sized irregular cribriform glands (modified from Ross et $a l^{9}$ ). Original magnification, 10x.

consecutive prostate cancers sent to this author over 7 months; 30 needle biopsy cases were selected that possibly represented cribriform Gleason pattern 3 cancer. ${ }^{10}$ Thirty-six digital images were taken and sent to 10 experts in prostate pathology with a consensus defined when at least 7/10 experts agreed 
on the grade. Even in this highly selected set of images thought to be the best candidates for cribriform pattern 3 from a busy consult service, most experts interpreted the cribriform patterns as pattern 4 . Conceptually, one would expect the change in grade from patterns 3 to 4 to be reflected in a distinct architectural paradigm shift where cribriform as opposed to individual glands are formed, rather than merely a subjective continuum of differences in size, shape, and contour of cribriform glands.

The only reason why cribriform pattern 3 even exists is because of the original Gleason schematic diagram. Gleason never specifically published the prognostic difference between what he called cribriform Gleason pattern 3 compared with Gleason pattern 4. Many of Gleason's cribriform Gleason pattern 3 cancers may not even have been infiltrating carcinomas due to the lack of availability of immunohistochemistry for basal cell markers. Today we might have diagnosed them either as cribriform high-grade PIN or intraductal carcinoma of the prostate (concepts not present in Gleason's era). Subsequently, there have been several studies demonstrating the adverse prognosis associated with cribriform glands. ${ }^{11-13}$ Based on all the above data, it was accepted in the 2014 grading conference that all cribriform cancer should be interpreted as Gleason pattern 4 and not pattern 3 .

\section{Gleason Pattern 4}

In 2005 it was accepted that ill-defined glands with poorly formed glandular lumina also warrant the diagnosis of Gleason pattern 4. Only a cluster of such glands, where a tangential section of Gleason pattern 3 glands cannot account for the histology, would be acceptable as Gleason pattern 4. In most cases, illdefined glands with poorly formed glandular lumina are accompanied by fused glands. Very small, well-formed glands still are within the spectrum of Gleason pattern 3.

\section{Glomeruloid Structures}

Glomeruloid glands in prostatic adenocarcinoma are characterized by dilated glands containing intraluminal cribriform structures with a single point of attachment, resembling a renal glomerulus (Figure 2a). ${ }^{14}$ On prostate biopsy, glomeruloid glands are exclusively associated with carcinoma and not associated with benign mimickers. A study of ours subsequent to the consensus conference indicated that glomerulations were overwhelmingly associated with concurrent Gleason pattern 4 or higher-grade carcinoma. ${ }^{15}$ In several cases, transition could be seen among small glomerulations, large glomeruloid structures, and cribriform pattern 4 cancer. Glomerulations represent an early stage of cribriform pattern 4 cancer and are best graded as Gleason pattern 4 .

\section{Gleason Pattern 5}

Gleason pattern 5 consists of individual cells, cords of cells, and sheets of tumor. Although typically one sees comedonecrosis with solid nests, occasionally one can see necrosis with cribriform masses that should be graded as Gleason pattern 5. One must be stringent as to the definition of comedonecrosis, requiring intraluminal necrotic cells and/or karyorrhexis, especially in the setting of cribriform glands. We have noted in two studies using different patient populations the tendency for pathologists to undergrade Gleason pattern 5 in almost $50 \%$ of cases sent for a second opinion at the request of the patient of urologist where this author has diagnosed Gleason pattern 5. ${ }^{16,17}$ Pattern 5 was missed more frequently when it was not the primary pattern.

\section{New approaches to display Gleason grades}

For the 2016 WHO Classification of Tumors of the Urinary System and Male Genital Organs, a revised schematic diagram was created with the assistance of David Grignon at the Indiana University School of

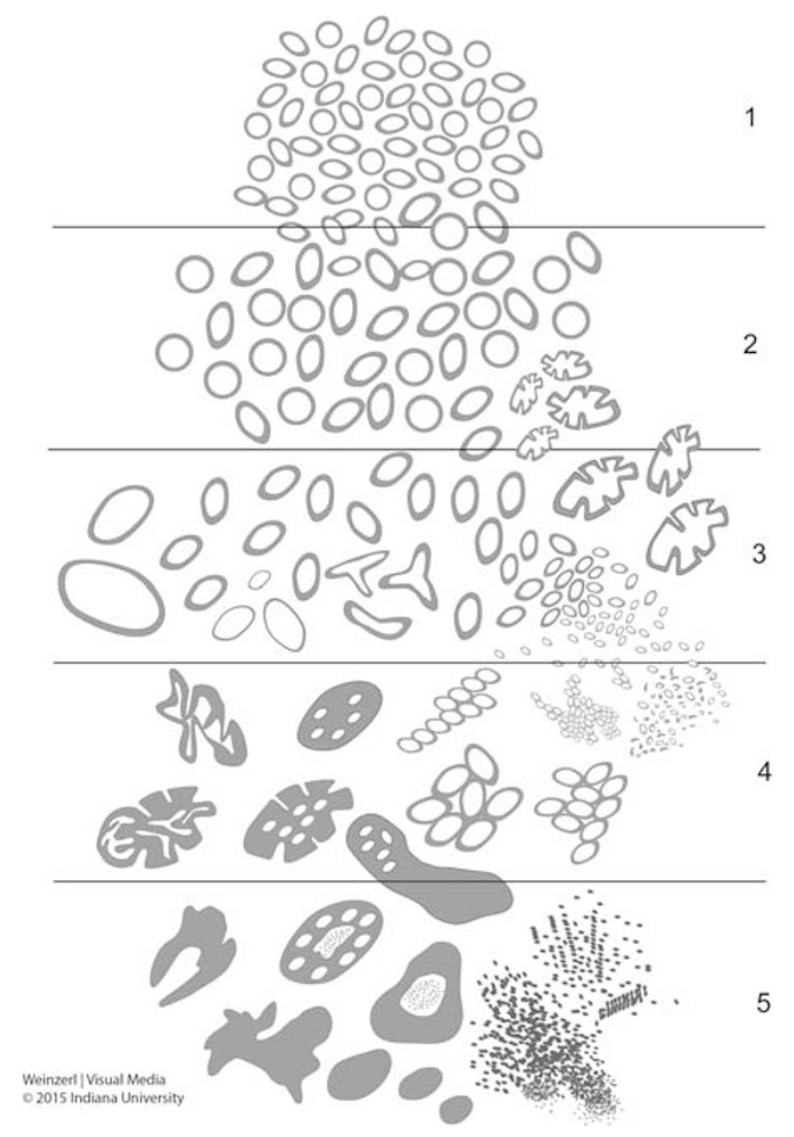

Figure 3 Current modified schematic Gleason diagram (courtesy of David Grignon, Indiana University Medical Center). 
Medicine (Figure 3). Advantages of the new schematic diagram is that it depicts variants of Gleason pattern 3 including pseudohyperplastic, atrophic, and branching prostate cancer.
Another tool that can aid in the practical grading of prostate cancer is a photomontage that shows multiple photomicrographs of various morphologies, both classic and difficult to grade, covering Gleason patterns

Discrete Well-formed Glands (Gleason Pattern 3)
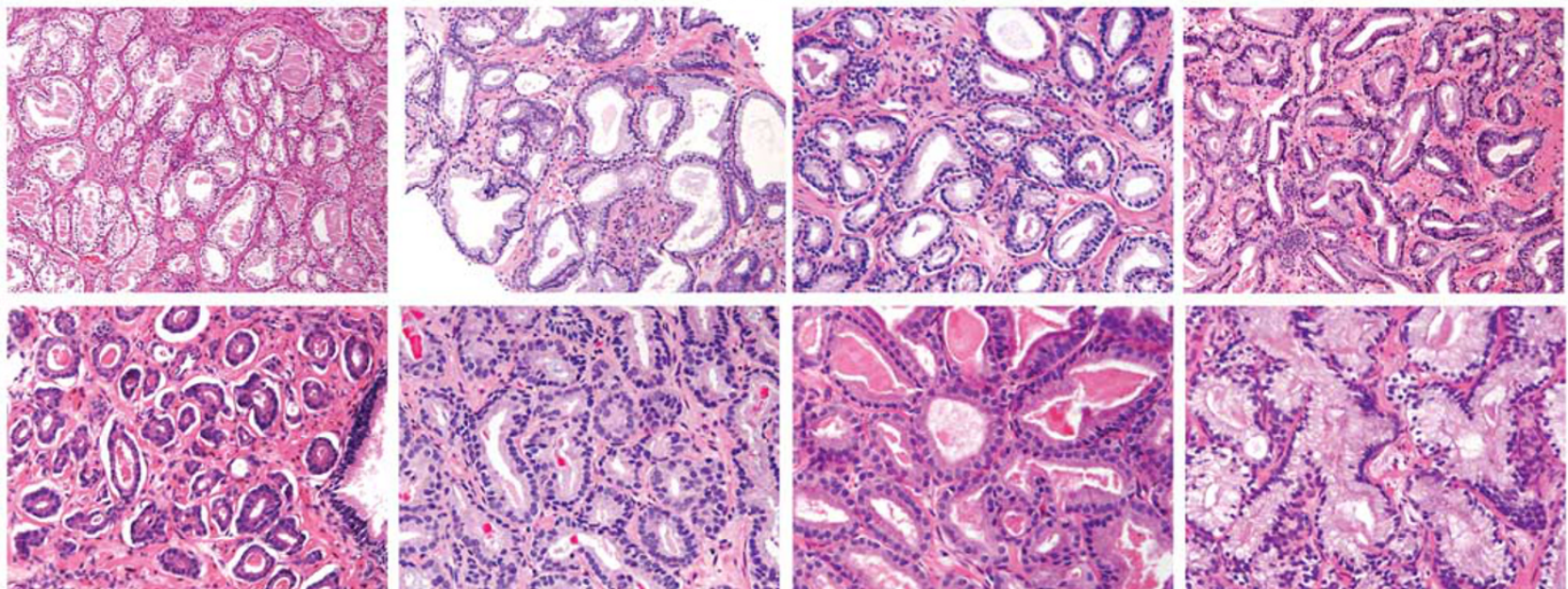

Cribriform/Poorly-formed/Fused Glands (Gleason Pattern 4)
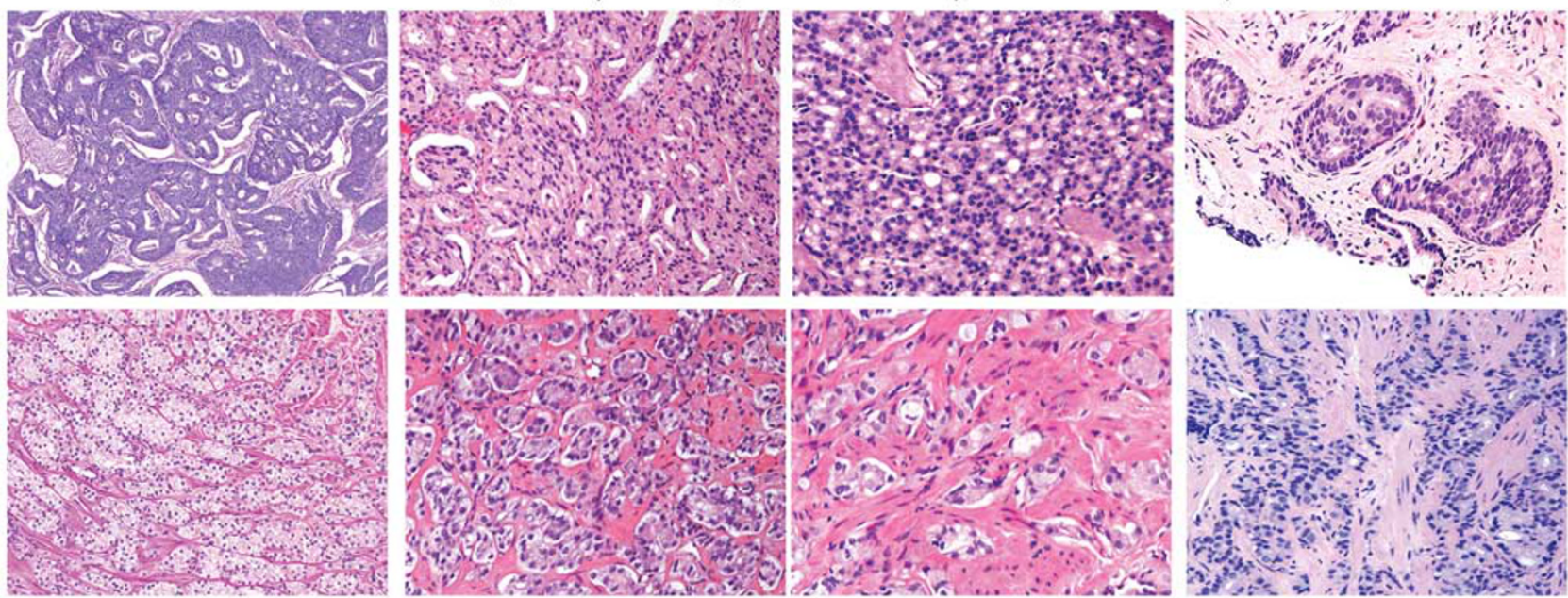

Sheets/Cords/Single Cells/Solid Nests/Necrosis (Gleason Pattern 5)
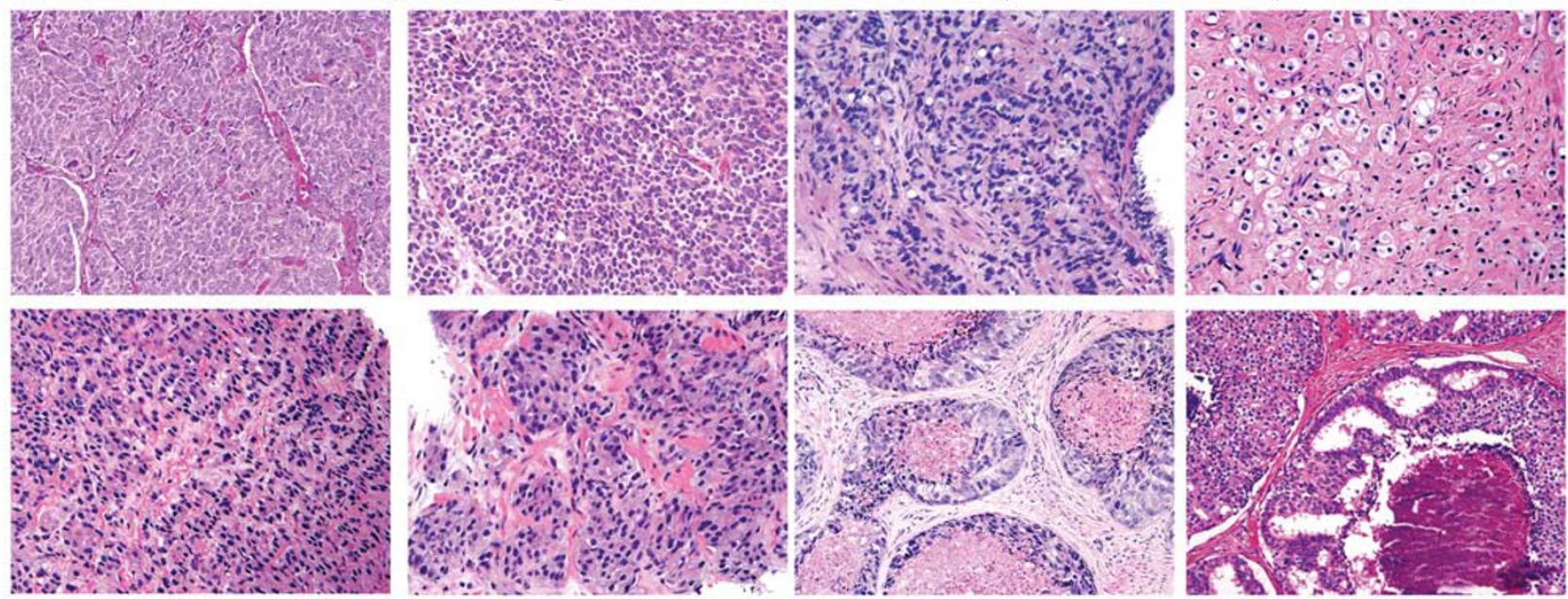

Jonathan Epstein 
3-5. It does not cover Gleason patterns 1 and 2, as in practice these are no longer in use (Figure 4). ${ }^{18}$

\section{Grading variants and variations of acinar adenocarcinoma}

A general rule that applies to variants, with the exception of small-cell carcinoma, is that variants should be graded, just the same as usual prostate adenocarcinoma, by evaluating the underlying architectural pattern.
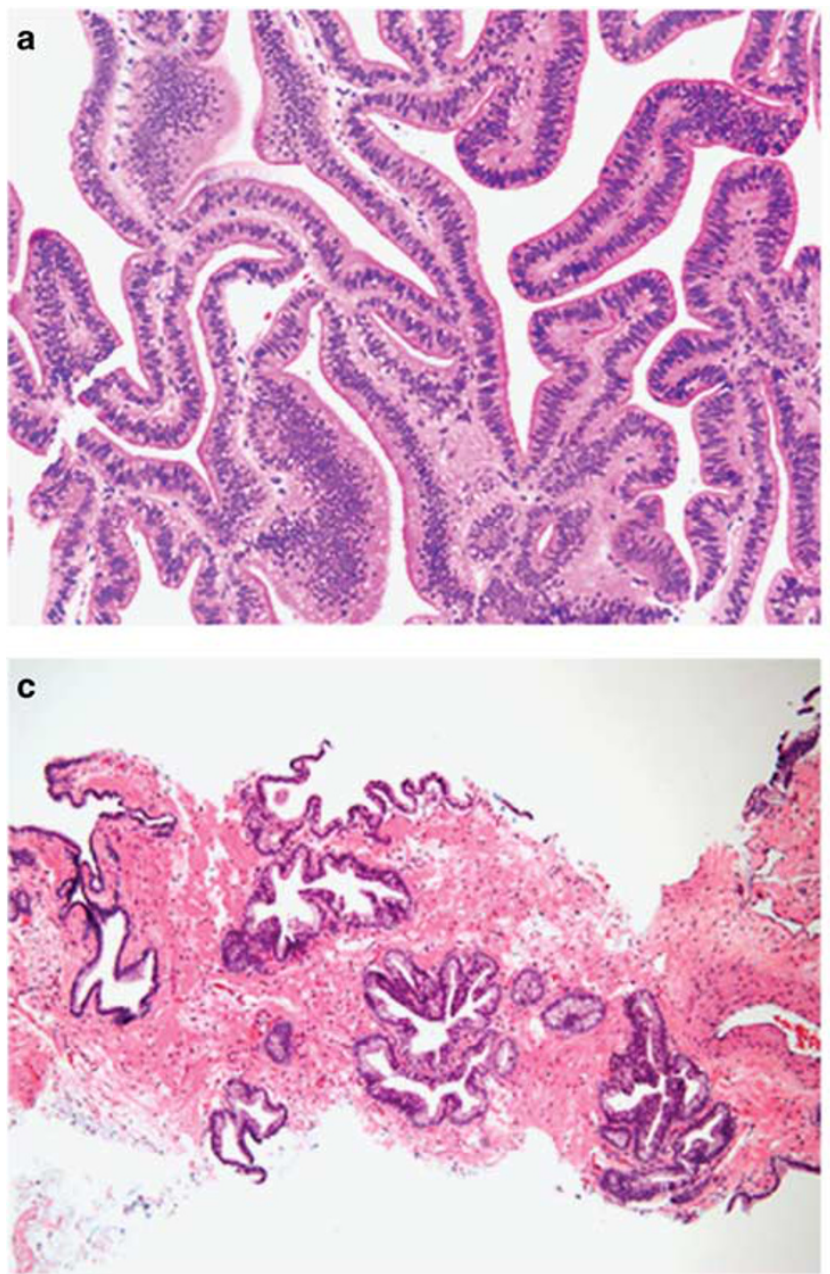

\section{Vacuoles}

Adenocarcinomas of the prostate may contain clear vacuoles and these should be distinguished from true signet-ring carcinomas which contain mucin. Whereas vacuoles in adenocarcinoma of the prostate are not uncommon, true mucin-positive signet-ring cell carcinomas of prostate are exceedingly rare with only a handful of bona fide cases reported in the literature. Vacuoles may distort the architecture and it is controversial as to what grade should be assigned. Gleason's only mention of vacuoles
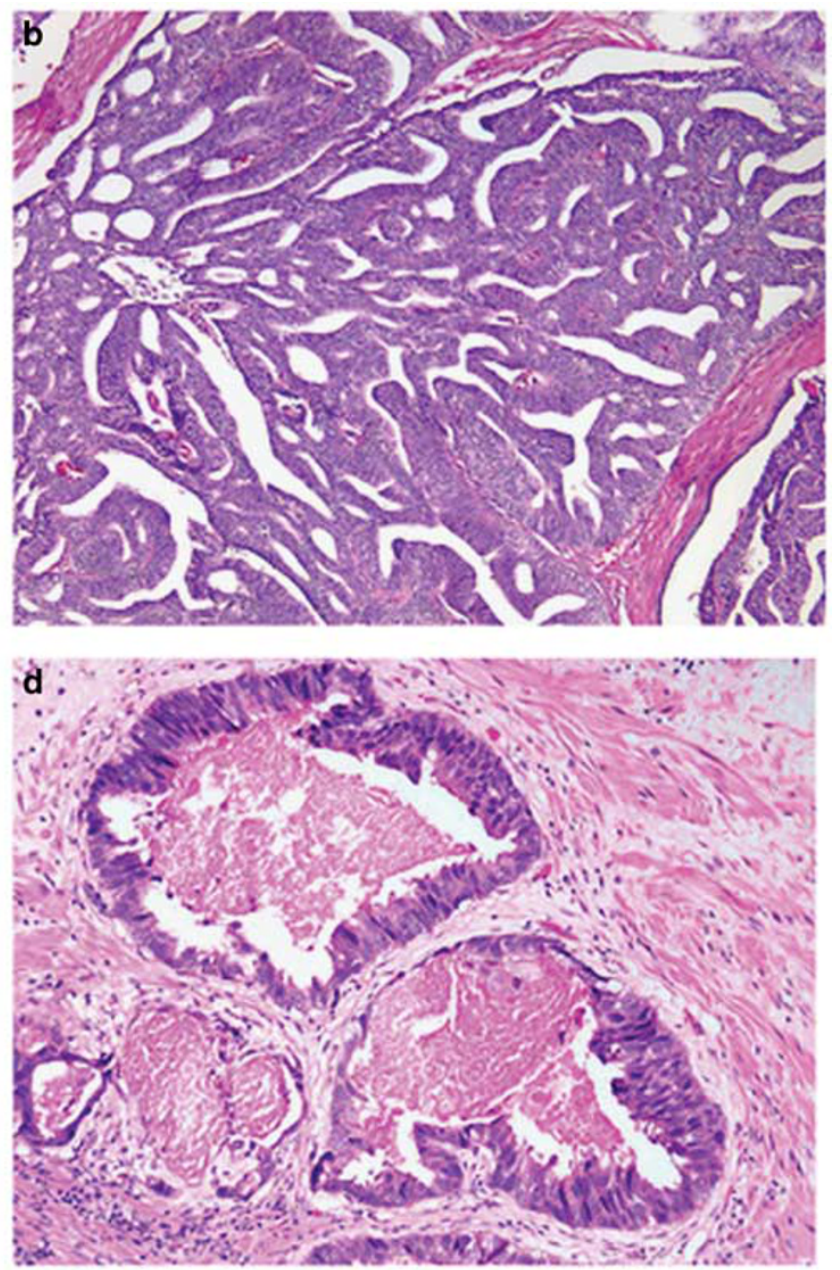

Figure 5 Prostatic ductal adenocarcinoma. (a) Papillary, Gleason pattern 4, original magnification, 20x. (b) Cribriform, Gleason pattern 4, original magnification, 10x. (c) PIN-like, Gleason pattern 3, original magnification, 10x. and (d) with necrosis, Gleason pattern 5, original magnification, 20x.

Figure 4 From left to right: (from reference 18). 1st Row: Closely packed uniform-sized and -shaped large glands. Large variably sized and shaped glands, some with infolding. Uniform medium-sized glands. Variably sized glands. 2nd Row: Occasional tangentially sectioned glands among well-formed small glands. Occasional tangentially sectioned glands among well-formed glands with open lumina. Back-toback discrete glands. Branching glands. 3rd Row: Large irregular cribriform glands with well-formed lumina. Irregular cribriform glands with slit-like lumina, glomeruloid structures, and fused glands. Irregular cribriform glands with small round lumina. Small round cribriform glands. 4th Row: Poorly formed glands with peripherally arranged nuclei. Small poorly formed glands. Small poorly formed glands. Fused poorly formed glands. 5th Row: Sheets of cancer. Sheets of cancer with rosette formation. Small nests and cords of tumor with scattered clear vacuoles. Individual cells. 6th Row: Nests and cords of cells with only vague attempt at lumina formation. Solid nests of cancer. Solid nests with comedonecrosis. Cribriform glands with central necrosis. 
described them as signet cells under pattern 5 tumor. Although typically vacuoles are seen within Gleason pattern 4 cancer, it may be seen within Gleason pattern 5 and even Gleason pattern 3 tumors. Tumors should be graded, as if the vacuoles were not present, by only evaluating the underlying architectural pattern.

\section{Foamy Gland Carcinoma}

In an analogous manner to handling cancers with vacuoles, in grading foamy gland carcinomas one should ignore the foamy cytoplasm and grade the tumor solely based on the underlying architecture. Whereas most cases of foamy gland carcinoma would be graded as Gleason score $3+3=6$, highergrade foamy gland carcinomas exist and should be graded accordingly based on the pattern. ${ }^{19,20}$

\section{Ductal Adenocarcinoma}

Ductal adenocarcinomas of the prostate most commonly are composed of either papillary fronds or cribriform structures. ${ }^{21}$ Ductal adenocarcinomas are recognized as being aggressive tumors with most studies showing comparable behavior to acinar cancer with a Gleason score $4+4=8$. Papillary and cribriform and ductal adenocarcinomas should be graded as Gleason score $4+4=8$, while retaining the diagnostic term of ductal adenocarcinoma to denote their unique clinical and pathological findings (Figure 5). This can be achieved by diagnosing such a tumor as 'prostatic ductal adenocarcinoma (Gleason score $4+4=8$ ).' In cases with mixed ductal and acinar patterns, the ductal patterns should be assigned Gleason pattern 4. The only exceptions to grading ductal adenocarcinoma as Gleason pattern 4 are: (1) PIN-like ductal adenocarcinoma; and (2) ductal adenocarcinoma with comedonecrosis. PIN-like ductal adenocarcinoma consists of individual glands lined by tall pseudostratified columnar cells resembling high-grade prostatic intraepithelial neoplasia. ${ }^{22}$ Its prognosis appears comparable to Gleason pattern 3. Although it has not been specifically studied, ductal adenocarcinoma with comedonecrosis is graded as Gleason pattern 5.

\section{Colloid (Mucinous) Carcinoma}

The majority of cases with colloid carcinoma consist of irregular cribriform glands floating within a mucinous matrix. ${ }^{23,24}$ These cases would be scored Gleason score $4+4=8$ (Figure 2e). The excellent prognosis of mucinous carcinomas in two large studies of mucinous carcinoma at RP supports grading mucinous prostate carcinomas based on the underlying architectural pattern rather than assuming that all of these tumors are aggressive. ${ }^{7,25,26}$

\section{Small-Cell Carcinoma}

Small-cell carcinoma of the prostate has unique histological, immunohistochemical, and clinical features. ${ }^{27}$ Comparable to its more common pulmonary counterpart, chemotherapy is the mainstay of therapy for prostatic small-cell carcinomas. These clinicopathologic features differ from those associated with Gleason pattern 5 prostatic acinar carcinoma, such that small-cell carcinoma should not be assigned a Gleason grade.

\section{Mucinous Fibroplasia (Collagenous Micronodules)}

The delicate ingrowth of fibrous tissue seen with mucinous fibroplasia can result in glands appearing to be fused resembling cribriform structures, although the underlying architecture is really that of individual discrete rounded glands invested by loose collagen. The tumor should be graded on the underlying glandular architecture, whereby the majority are graded as Gleason score $3+3=6 .{ }^{14}$ Only when there are distinct cribriform glands in areas of mucinous fibroplasias does this author diagnose Gleason pattern 4.

\section{Pseudohyperplastic Adenocarcinoma}

Uncommonly, adenocarcinomas of the prostate share some architectural features with benign glands, including larger size, branching, and papillary infolding. These cancers should be graded as Gleason score $3+3=6$ with pseudohyperplastic features. ${ }^{28,29}$ This convention is in large part based on the recognition that they are most often accompanied by more ordinary Gleason score $3+3=6$ adenocarcinoma.

\section{Post-Treatment Cancer: Hormone Therapy and Radiation Therapy}

If histologically ordinary prostate cancer is seen, which resembles non-treated cancer, the diagnosis of 'Cancer without significant treatment affect' is given and a Gleason grade is assigned. If cancer is present, yet shows treatment affect, then the diagnosis is 'Cancer with significant treatment affect' and a grade is not assigned.

\section{Reporting rules for Gleason grading reporting secondary patterns of higher grade when present to a limited extent}

High-grade tumor of any quantity on needle biopsy, as long as it was identified at low to medium magnification (see General Applications of the Gleason Grading System) should be included within the Gleason score. Any amount of high-grade tumor sampled on needle biopsy most likely indicates a 
more significant amount of high-grade tumor within the prostate due to the correlation of grade and volume and the problems inherent with needle biopsy sampling. Consequently, a needle biopsy that is entirely involved by cancer with 98\% Gleason pattern 3 and $2 \%$ Gleason pattern 4 would be diagnosed as Gleason score $3+4=7$.

In RP specimens with the analogous situation of a tumor nodule having $98 \%$ Gleason pattern 3 and $2 \%$ pattern 4 , the current recommendation is to grade these foci in an analogous manner to that done on needle biopsy and interpret the case as Gleason score $3+4=7$ and record the percentage of pattern 4 .

The prognostic significance of minor high-grade patterns in RP specimens were first analyzed in 2000 by Pan et al, ${ }^{30}$ who used the term tertiary grade pattern. ${ }^{30}$ The original Gleason grading system did not account for more than two patterns. At the 2014 meeting, a review of the literature was presented summarizing studies on tertiary patterns where at least $50 \mathrm{RP}$ specimens were analyzed. Of the eight articles that fulfilled this criteria, seven reported that tertiary grade patterns adversely affected prognosis. ${ }^{31-38}$ A conflicting study by Isbarn et al ${ }^{39}$ found that tertiary patterns $<5 \%$ was not associated with adverse prognostic findings at RP, although a potentially confounding factor in this study was the tertiary lower patterns (ie Gleason score $3+4=7$ with tertiary pattern 2) was considered tertiary grade patterns, whereas most other studies evaluated only tertiary higher-grade patterns. ${ }^{39}$ Based on these data, there was a consensus that tertiary grade patterns as an important prognostic factor. Subsequent to the 2014 meeting, there has been another large study on this topic further supporting the adverse prognostic significance of higher tertiary grade patterns. ${ }^{40}$

One of the confusing issues with the term tertiary grade pattern as it has been used in the past and still persists today is the use of the term tertiary for RP tumors sometime referred to cases with only two grade patterns, where the secondary higher-grade pattern was of very limited extent. For example, some pathologists grade RPs with $3+3=6$ and $<5 \%$ pattern 4 as $3+3=6$ with tertiary 4 , to contrast with $5-50 \%$ pattern 4 , which they grade as $3+4=7 .{ }^{36}$ Using tertiary in this context, despite there not being three different grade patterns, accounts for the less adverse prognosis with very limited pattern 4 relative to greater amounts of pattern 4 . With the adoption of reporting percentage Gleason pattern 4 in Gleason $3+4=7$ as noted below, cases formerly reported as Gleason score $3+3=6$ with tertiary pattern 4 will now be recorded as Gleason score 3 $+4=7$ with $<5 \%$ pattern 4 . Similarly, some pathologists graded tumors composed of $>95 \%$ Gleason pattern 4 and $<5 \%$ Gleason pattern 5 as Gleason score $4+4=8$ with tertiary pattern $5 .{ }^{36}$ Recent studies have shown that presence of limited ( $<5 \%$ ) Gleason pattern 5 in the context of Gleason score $4+4=8$ imparts a poor prognosis equivalent to Gleason scores 9-10. ${ }^{41}$ Although not discussed at the 2014
Consensus Conference, a collaborative study of 169 RPs performed at the Johns Hopkins Hospital and University of Pittsburgh has shown that the presence of $<5 \%$ Gleason pattern 5 in the context of Gleason score $4+4=8$ results in a biochemical risk-free survival that is equivalent to Gleason scores 9 and 10. ${ }^{41}$ At the 2014 Consensus Conference, there was agreement that the grading rule proposed in the 2005 Consensus Conference that on needle biopsies, tertiary is not used and rather the most common and highest grade patterns are summed together as the Gleason score. ${ }^{6}$ For example, in a needle biopsy core with $70 \%$ Gleason pattern $3,25 \%$ pattern 4 and $5 \%$ pattern 5 , the tumor would be graded as Gleason score $3+5=8$.

Another controversial aspect of the term tertiary grade patterns is whether there is an upper limit to the amount of Gleason pattern 5 that can still be considered as a tertiary pattern. The first article to investigate the prognostic significance of tertiary patterns from the Johns Hopkins Hospital referred to tertiary pattern 5 as 'very limited amounts of pattern $5 ' .{ }^{30}$ Subsequent works on this topic from the same institution more precisely restricted the use of 'tertiary pattern' to prostate adenocarcinoma at RP with $<5 \%$ pattern $5 .{ }^{36}$ There is, however, a lack of consensus on whether there should be a minimum cutoff for the amount of percent pattern 5 in cases when pattern 5 is the third most common Gleason pattern. Choy et $a l^{42}$ and Lucca et $a l^{40}$ required $<5 \%$ Gleason pattern 5 for it to be considered tertiary pattern 5 , but Adam et al ${ }^{33}$ only required that pattern 5 be the third most common pattern, regardless of percentage. An argument for restricting the amount of percent pattern 5 to be designated as 'tertiary pattern 5 ' is that one could have, for example, an RP tumor nodule with $50 \%$ pattern 3 , $30 \%$ pattern 4 , and $20 \%$ pattern 5 . With no limits on how much pattern 5 is allowed for a tertiary pattern, this tumor could be graded as Gleason score $3+4=7$ with tertiary pattern 5. Gleason score $3+4=7$ with tertiary pattern 5 is typically considered only Gleason score $3+4=7$ for treatment purposes. A Gleason score $3+4=7$ at RP has almost a $90 \%$ cure rate, which would give the misleading impression of a very favorable tumor, given that the tumor had $30 \%$ pattern 4 and $20 \%$ pattern 5. At the 2014 Consensus Meeting, $83 \%$ of participants favored the term of minor high-grade pattern over tertiary grade pattern. 'Minor' indicates that the high-grade pattern should be limited in extent, and not just the third most common pattern. The only cutoff that has been used in the literature with evidence-based data correlating with outcome has been $<5 \%$ for tertiary pattern 5. The term tertiary or minor high-grade pattern should only be used in the logical scenario when there are 3 grade patterns, such as with $3+4=7$ or $4+3=7$ with $<5 \%$ Gleason pattern 5 at RP.

Minor (tertiary) high-grade patterns do not change the Grade Groups (ie Gleason score 3+4=7 (Grade Group 2) with minor (tertiary) pattern 5). If Grade 
Groups 1-5 eventually were to replace Gleason scores 2-10, the 2014 Consensus Conference suggested options how to incorporate tertiary patterns with Grade Groups. Suggestions included, for example, Grade Group 2 with minor high-grade pattern or Grade Group $2^{+} .43$

In summary, the term 'tertiary' should only be used when there are three patterns with a very minor component of higher grade. On an RP, if pattern 5 is $<5 \%$ and the third most common pattern then it should be reported as $3+4=7$ with minor high-grade (tertiary) pattern 5 . If pattern 5 is $>5 \%$ then it is the secondary pattern (ie. $3+5=8$ ). On needle biopsy, if pattern 5 is the $3^{\text {rd }}$ most common pattern, regardless of percentage, then it is included in the Gleason score (ie $3+5=8$ ) (most common+highest grade). Only use the term 'minor high-grade (tertiary) pattern' for Gleason score $3+4=7$ with $<5 \%$ pattern 5 or Gleason score $4+3=7$ with $<5 \%$ pattern 5 on RP. ${ }^{43}$

\section{Reporting secondary patterns of lower grade when present to a limited extent}

In the setting of high-grade cancer one should ignore lower-grade patterns if they occupy $<5 \%$ of the area of the tumor. For example, a needle biopsy core that is $100 \%$ involved by cancer, with $98 \%$ Gleason pattern 4 and $2 \%$ Gleason pattern 3 would be diagnosed as Gleason score $4+4=8$. These cases with extensive pattern 4 cancer, where a significant amount of tumor is available for examination, should be considered as high grade (Gleason score $\geq 8$ ). At the other extreme, one can occasionally see small foci of Gleason pattern 4 on needle biopsy with a few glands of pattern 3 . In the setting of very limited cancer on needle biopsy, the few glands of pattern 3 would typically occupy over $5 \%$ of the area of the tumor focus, and one would grade these tumors as Gleason score $4+3=7$. The same $5 \%$ cutoff rule for excluding lower-grade cancer also applies for TURPs and RP specimens, which in most cases would relate to extensive cancer with $>95 \%$ Gleason pattern 4 tumor.

\section{Reporting percentage pattern 4}

A major new recommendation that came from the 2014 Consensus Conference, subsequently supported by the $2016 \mathrm{WHO}$, was to report percent pattern 4 with Gleason score 7 in both needle biopsies and RP specimens. ${ }^{43,44}$ This recommendation was supported by $75 \%$ of the conference pathologists and $100 \%$ of the clinicians, with an overall consensus of $79 \%$.

The major advantage to report percent pattern 4 is for patient care. ${ }^{43}$ Currently, active surveillance is typically restricted for men with Gleason score 3 $+3=6$. However, some urologists could consider this strategy for men with Gleason score $3+4=7$ with limited percent pattern 4, especially in older men, or with increased comorbidity, or where cancer appears relatively small based on the extent of cancer on biopsy and/or imaging studies. ${ }^{45,46}$ Recording the percent pattern 4 would also potentially be helpful in cases that are borderline between Gleason score 3 $+4=7$ or Gleason score $4+3=7$, as there are different radiation therapy approaches for Gleason score 3 $+4=7$ (Grade Group 2) versus 4+3=7 (Grade Group 3). ${ }^{47}$ By reporting the case as $3+4=7$ (approaching $50 \%$ pattern 4$)$ or $4+3=7(60 \%$ pattern 4$)$, the borderline nature of the case would be evident and clinicians could use other factors for guiding therapy.

Although recording the percent pattern 4 introduces an extra step in the evaluation of prostate specimens, it is relatively minimal. Pathologists have to decide what part of the tumor is pattern 4 or 3 when assigning a Gleason score so that estimating percent pattern 4 is not that much extra effort. Assessing the percent Gleason pattern 4 is relatively reproducible, with substantial agreement within $\pm 10 \%$ in cases. ${ }^{48}$ Cases where patterns 3 and 4 are intermingled are more difficult to assess for percent pattern 4. ${ }^{49}$ We have shown that there is only moderate interobserver agreement for assessing the percent pattern 4 with $<10 \%$ involvement of the core by cancer, as a few glands of a given pattern can markedly affect the percent of Gleason pattern $4 .{ }^{48}$ As a result, some experts do not record percent pattern 4 in small foci of Gleason score 7 tumors on needle biopsy, although other experts routinely reports percent pattern 4 for call cases of Gleason score regardless of tumor extent.

It was the majority opinion of the 2014 Consensus Meeting that the method by which the percent pattern 4 should be recorded remains optional, both for the core and case level. One option is to record the percentages as follows: $<5,10,20,30$, and $40 \%$, approaching 50\% for Gleason score $3+4=7$, and 60 , 70,80 and $90 \%$ for Gleason score $4+3=7$, which is the personal preference of the current author. Another alternative would be $<5 \%, 5-<25 \%, 25-$ $<50 \%, 50-<75 \%$, and 75-90\%. Given the inherent large sampling error with needle biopsies, it does not make sense to record more precise percentages (ie $13 \%$ ). Although not discussed at the 2014 Consensus meeting, some experts do not record percent of Gleason pattern 4 if any other core has Gleason score 9 or 10 (Grade Group 5), as the latter grades drive therapy and prognosis. ${ }^{43}$ Although not discussed at the 2014 Consensus meeting, it is the personal preference of this author and several other urological pathology experts to record percent pattern 4 for those cores with Gleason score 7 if the highest core is Gleason score $4+4=8$, as it can still provide useful information for the clinician. ${ }^{43}$ For example, if in addition to a core with Gleason score $4+4=8$, there are other cores with $4+3=7$, with $80-90 \%$ pattern 4 that informs the clinician that the overall tumor grade is closer to a Gleason score 8. 
Subsequent to the 2014 Consensus Conference, studies have demonstrated that increasing percent pattern 4 at RP correlates with an increased risk of biochemical recurrence after RP. ${ }^{42,50,51}$ There have also been several publications showing that percent pattern 4 on needle biopsy can improve prediction of upgrading at RP and of adverse findings at RP and biochemical recurrence after RP. ${ }^{52,53}$

\section{General applications of the Gleason grading system}

As described by Gleason, the initial grading of prostate carcinoma should be performed at low magnification using a $\mathrm{x} 4$ or $\mathrm{x} 10$ lens. After one assesses the case at scanning magnification, one may proceed to use the x20 lens to verify the grade. For example, at low magnification one may have the impression of fused glands or necrosis, but may require higher magnification at $\mathrm{x} 20$ to confirm its presence. However, one should not initially use the $\mathrm{x} 20$ or $\mathrm{x} 40$ objectives to look for rare fused glands or a few individual cells seen only at higher power, which would lead to an overdiagnosis of Gleason pattern 4 or 5, respectively (Figure 6). If the tumor is borderline between lowerand higher-grade cancer, I assign the lower grade so as to not result in overtreatment. In cores with borderline grade, additional levels are often helpful to clarify the grade.

\section{Needle biopsy with different cores showing different grades}

A controversial area discussed at the 2014 Consensus conference is how to handle cases where multiple cores with different Gleason scores are positive for cancer. Should the overall grade be the core with the highest grade or does one assign the grade by

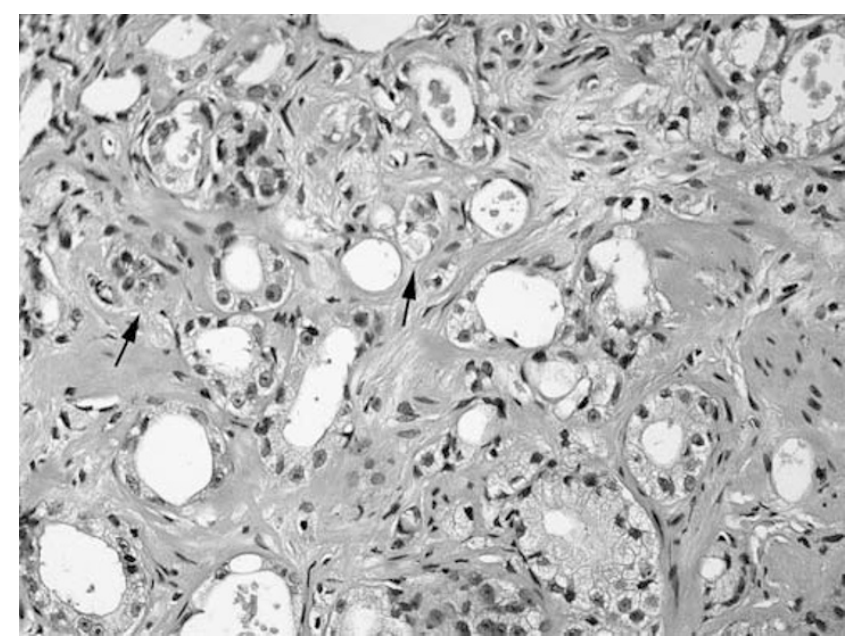

Figure 6 Gleason pattern 3 with a few glands (arrows) that appear poorly formed but are likely tangential sections off of adjacent small well-formed glands. Original magnification, 40x. mentally adding all the cancer together as if it was one long core? Whether the highest grade per core or the overall score is used impacts a significant number of cases. ${ }^{54}$ Most studies show that the highest Gleason score on a given core correlates better with stage and Gleason score at RP than the average or most frequent grade among the cores. ${ }^{55-58}$ Fewer studies show no significant difference in biochemical recurrence and prostate cancer death whether recording as the case grade either the higher Gleason score or the average Gleason score. ${ }^{59,60}$ All of the commonly used tables (ie Partin tables) and nomograms (Kattan nomograms) to predict prognosis and stage have used the highest core grade in the case. ${ }^{61,62}$ In general, clinicians use the highest Gleason score per part in a case for prognosis and therapeutic purposes, which was also the viewpoint of clinicians attending the 2014 Consensus Conference. The problem with using the average grade is that when there are multiple cores with different grades, it can be difficult and subjective to determine if one is dealing with two separate tumors with differing grades versus a single tumor with grade heterogeneity. At the 2014 Consensus Conference, $65 \%$ of pathologists and clinicians stated that the highest grade core should be used for clinical decision making and $10 \%$ favored using the global grade for the entire case $(25 \%$ said either option was acceptable). Only $3 \%$ of the experts at the 2014 Consensus Conference recommended reporting only the average or global grade for the entire case with approximately one-quarter of them providing the overall grade in addition to the individual core grade, both practices more commonly seen outside the United States. The remaining majority of the pathologists at the 2014 Consensus Conference report out the individual Gleason scores per part, which is standard practice in the United States.

When multiple cores having different grades are present in the same specimen container, the recommendation at the 2014 Consensus Conference was equally split between assigning a grade to each positive core versus a global grade for each specimen container, with the same conclusion as the 2005 consensus: Assign individual Gleason scores to separate cores as long as the cores were submitted in separate containers or the cores were in the same container yet specified by the urologist as to their location (ie by different color inks). In cases where there are different undesignated cores with different grades in the same specimen container, it is optional whether to assign individual grades to different cores or a global grade for the specimen container. In addition to giving separate cores individual Gleason scores, one has the option to also give an overall score at the end of the case. ${ }^{6}$ As currently cores are almost always submitted per part based on a specific site (ie not just left versus right), one can assume that all the cores in a given part (if not inked differentially) came from roughly the same location within the prostate so that only one grade averaging all the 
positive cores per part should be reported. In cases with multiple fragmented cores in a jar, there was a $97 \%$ agreement to give a global Gleason score for that jar. For example, diagnosing Gleason score $4+4=8$ on a tiny tissue fragment where there are other fragments with a greater amount of Gleason pattern 3 could be in error; if the cores were intact and the tumor was all on one core, it would be assigned a Gleason score $3+4=7$.

\section{RP specimens with separate tumor nodules}

RP specimens should be processed in an organized manner where one can make some assessment as to whether one is dealing with a dominant nodule or separate tumor nodules. This does not necessarily require serially sectioning and embedding an RP in its entirety. Rather, multiple sampling techniques have described how one can subtotally submit the prostate yet still maintain orientation to distinguish between different tumor nodules. ${ }^{63-65}$ This issue becomes critical in the situation where one has a higher-grade peripheral nodule and a smaller, typically transition zone, lower-grade nodule. One can have a nodule of Gleason score $4+4=8$ within the peripheral zone and a Gleason score $2+2=4$ nodule within the transition zone. Occasionally these Gleason score $2+2=4$ transition zone tumors may even reach relatively sizable proportions, although typically they are organ confined. If one were to assign an overall score considering all of the tumor within the prostate as one lesion, the score of such a tumor would be Gleason score 4 $+2=6$ or Gleason score $2+4=6$. Such a grade would be misleading as it is not logical to expect that the presence of a lower-grade tumor that is discrete from a separate high-grade tumor nodule could in some way mitigate the poor prognosis associated with the higher-grade tumor nodule. One should assign a separate Gleason score to each dominant tumor nodule(s). Most often, the dominant nodule is the largest tumor, which is also the tumor associated with the highest stage and highest grade. In the unusual occurrence of a non-dominant nodule (ie smaller nodule) that is of higher stage, one should also assign a grade to that nodule. If one of the smaller nodules is the highest grade focus within the prostate, the grade of this smaller nodule should also be recorded. In general, this will be the exception; in most cases, separate grades will be assigned to only one or at most two dominant nodules.

\section{A new grading system for prostate cancer}

\section{Should Gleason Score 6 Cancer be Renamed as Non-Cancer}

Overtreatment of low-grade prostate cancer (Gleason score $\leq 6)$ is a recognized problem today with systematic prostate gland sampling triggered by PSA measurements. Fear of death from cancer likely has some role, and removing the label 'cancer' could reduce unnecessary treatment of low-grade disease. ${ }^{66}$ Two studies showing that using a contemporary grading approach, pure Gleason score 3 $+3=6$ at $\mathrm{RP}$ is incapable of regional lymph node metastasis. $^{9,67}$ At RP, pure Gleason score $3+3=6$, organ-confined, margin-negative disease has an excellent prognosis with only occasional men demonstrating detectable PSA. ${ }^{68,69}$ As a result of, it has been questioned if Gleason score $3+3=6$ should retain the designation of cancer or be relabeled as indolent lesion of epithelial origin (IDLE) to avoid fear and consequential overtreatment of a proportion of potentially indolent prostate cancers. ${ }^{70}$ Although renaming Gleason score 6 adenocarcinoma as 'not cancer' seems farfetched, there is a precedent in other organ systems to rename indolent malignancies with a non-cancerous term to avoid overtreatment. Some examples include: (1) renaming welldifferentiated liposarcoma in the extremities as atypical lipomatous tumor; ${ }^{71}$ (2) changing the designation of a subset of bladder tumors previously diagnosed as low-grade papillary urothelial carcinoma to papillary urothelial neoplasm of low malignant potential; ${ }^{72}$ and (3) most recently reclassifying encapsulated follicular variant papillary thyroid carcinoma to noninvasive follicular thyroid neoplasm with papillary-like nuclear features. ${ }^{73}$

From a pathologist's viewpoint, Gleason score 6 is still cancer with many of the same morphological features of higher-grade cancer, along with a lack of a basal cell layer and the potential to locally invade. ${ }^{74}$ Gleason pattern 3 cancer harbors many of the molecular alterations associated with higher-grade cancers including overexpression of $\alpha$-methylacylCoA racemase, glutathione $S$-transferase hypermethylation and downregulation, and TMPRSS2: ERG gene fusions. ${ }^{75}$ From a clinical perspective, renaming Gleason score $3+3=6$ as an IDLE tumor on biopsy carries the risk that patients on active surveillance will not adhere to long-term follow-up as they have been told they do not have cancer. The need for close follow-up results from the risk of unsampled higher-grade carcinoma. The likelihood of upgrading from a Gleason score $\leq 6$ on biopsy to Gleason score $\geq 7$ at RP has been reported to be as high as $36 \%{ }^{76}$ If Gleason score 6 on biopsy was not labeled as cancer, the potential for higher grade or more extensive disease might be ignored, and compliance with recommendations for careful monitoring may not occur.

\section{Problem with the Current Gleason Scale}

Consequently, Gleason score 6 prostatic adenocarcinoma should still be called cancer. However, there is a need to change what patients think when they hear they have Gleason score 6 cancer. Urologists need to 
reassure and educate patients, and pathologists need to modify how we report prostate cancer grade to more accurately reflect their behavior. A major problem with the current application of the Gleason grading system is that Gleason score 6 is the lowest grade reported on biopsy, although the scale goes from 2-10. What other grading system starts with a 6 ? Patients are told they have a Gleason score of 6 out of 10 and logically but incorrectly think that they have a tumor in the middle of the grade spectrum, contributing to the fear of cancer. I routinely talk to patients on a daily basis regarding their pathology on prostate needle biopsy as it relates to treatment and prognosis. The first thing I tell them when describing their Gleason score 6 cancer is that it is the lowest possible grade. An anecdotal experience I had talking with a particular patient exemplifies the problem with the current Gleason scale. A man that I recently diagnosed as having Gleason score $3+4=7$ on biopsy called me, literally in tears. His wife was recently diagnosed with a glioblastoma and he feared that given that his prostate cancer grade was a 7 , which was close to 10 (the worst grade), he might not be alive to take care of his wife. I was able to reassure him that from his cancer's standpoint it has approximately an $88 \%$ cure rate and even if it did recur it would not do so symptomatically for many years. The power of how prostate cancer grades are reported was also recently shown in a study of prostate cancer patients in two clinical settings from 2015 to $2016 .{ }^{77}$ The men were told that there is a new grading system for prostate cancer with grades ranging from 1 to 5, where Grade Group 1 is equivalent to Gleason score 6 . The majority of patients (84\%) agreed that it would be clearer if grades were reported on a scale of 1-5 instead of 610. Eighty-eight percent of the men would prefer to hear they have 'Grade Group 1' rather than 'Gleason 6', and, most importantly, $80 \%$ would feel more comfortable choosing active surveillance with 'Grade Group 1' versus 'Gleason 6'.

\section{Problems with Gleason System Grouping}

In the literature and for therapeutic purposes, various Gleason scores have been incorrectly grouped together based on the assumption that they have a similar prognosis. Analyzing some of the highest impact articles on prostate cancer in the past few years reveals some of the Gleason score groupings that have been used: 2-4, 5-7, 8-10 (Prostate Cancer Outcomes Study); ${ }^{78} 2-6,7,8-10$ (Scandinavian Prostate Cancer Group Study); and 2-6, 7-10 (Prostate Cancer Prevention Trial and Prostate Cancer Intervention versus Observation Trial). ${ }^{79,80}$ The most common risk stratification used in prostate cancer is the D'Amico classification also used by the National Comprehensive Cancer Network. ${ }^{81}$ It stratifies prostate cancer based on serum PSA values, clinical stage, and biopsy score into low-, intermediate-, and high-risk groups incorporating Gleason scores into a three-tiered Gleason score grouping (2-6, 7, and 8-10). In addition to the lack of uniformity of the various score groupings, precluding meaningful comparisons between studies, the combinations used have significant flaws. Gleason scores 2-4 virtually never exist on current biopsy material. Many of these cases in Gleason's era, predating the use of modern immunohistochemistry, probably represent adenosis, a mimicker of cancer. Studies combining Gleason scores 6 and 7 span tumors with almost a uniformly excellent prognosis $(3+3)$ to those with a substantial likelihood of progressing following therapy $(4+3)$. In the former, many men are candidates for active surveillance, whereas in the latter typical treatment is either by surgery or combination androgen deprivation and radiation therapy. All of the above classification systems consider Gleason 7 as a single score without distinguishing $3+4$ versus $4+3$, despite studies, showing a significantly worse prognosis for the latter. ${ }^{82-84}$ Combining scores 7-10 includes cases with an excellent prognosis with an $88 \%$ cure rate (3 +4 ) along with those that have a high prostate cancerspecific lethality $(5+5)$. Even within the high score group of 8-10, we have demonstrated that Gleason scores 9-10 have twice as poor a prognosis than Gleason score 8, an observation supported by multiple studies noting the adverse prognosis associated with Gleason pattern $5 .^{85-91}$

\section{Proposal for a New Grading System}

Although Gleason scores range from 2 to 10, there are 25 potential scores $(1+1,1+2,1+3,1+4,1+5,2+1$, etc). If one were coming up with a new grading system starting from scratch one would want to distill it down to a simple system with the least number of grades, each with their distinct prognosis. Based on a series of 6462 men treated by RP where both the needle biopsy and RP were graded using the current modified Gleason grading system, this author showed both for biopsy and for RP that the following Gleason grade groups accurately reflects prognosis: Gleason score 2-6 (Prognostic Grade Group 1); Gleason score $3+4=7$ (Prognostic Grade Group 2); Gleason score 4+3=7 (Prognostic Grade Group 3); Gleason score 8 (Prognostic Grade Group 4); and Gleason scores 9-10 (Prognostic Grade Group 5). ${ }^{85}$

In a recent meta-analysis of over 20000 men treated by RP from five institutions, Grade Groups were strongly correlated with risk of biochemical recurrence after surgery. ${ }^{92}$ Assigning a risk of 1 to Gleason score 6 , the relative risks of progression for Grade Groups 2-5 were 2.6, 8.5, 16.8, and 29.3. The 5 -year biochemical risk-free survival was $97.5 \%$, $93.1 \%, 78.1 \%, 63.6 \%$, and $48.9 \%$ for Grade Groups 1-5, respectively. These grade grouping was also validated on biopsy correlating with risk of progression after RP and following radiation therapy. 
Table 1 Definition of Grade Groups

Grade Group $1(3+3=6)$-Only individual discrete well-formed glands

Grade Group $2(3+4=7)$-Predominantly well-formed glands with lesser component of poorly formed/fused/cribriform glands

Grade Group $3(4+3=7)$ —Predominantly poorly formed/fused/ cribriform glands with lesser component of well-formed glands ${ }^{\mathrm{a}}$

Grade Group 4 (Gleason score 8)

Only poorly formed/fused/cribriform glands ${ }^{\mathrm{a}}$ or

Predominantly well-formed glands and lesser component lacking glands ${ }^{\mathrm{b}}$

Predominantly lacking glands and lesser component of well-formed glands ${ }^{b}$

Grade 5 (Gleason scores 9 and 10)—Lack gland formation (or with necrosis) with or w/o poorly formed/fused/cribriform glands ${ }^{\mathrm{a}}$

${ }^{\mathrm{a}}$ For cases with $>95 \%$ poorly formed/fused/cribriform glands or lack of glands on a core or at RP, the component of $<5 \%$ well-formed

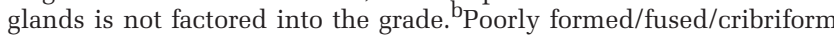
glands can be a more minor component.

Following these initial studies, there have been numerous works that have validated the new grading system following RP and radiation therapy, including its correlation with prostate cancer death and distant metastases. ${ }^{60,93-101}$

These new Grade Groups were formally accepted by the 2016 World Health Organization (WHO and it was accepted that at least for the foreseeable future, Grade Grouping would be report alongside the Gleason score (ie Gleason score 3+4 = 7 (Grade Group 2)), with eventually possibly replacing it in the future. The new system has also been accepted by the College of American Pathologists where it will be mandatory to report Grade Groups starting in 2018, and the AJCC Cancer Staging Manual 8th Edition. The histological definition of the five grades in the new grading system is depicted in Table 1 . While in the foreseeable future, one would think of the new Grade Groups in terms of their equivalence to the corresponding Gleason grade, if eventually Grade Groups replaces the Gleason system, then the definitions of the Grade Groups would be based on the descriptive text shown in Table 1.

There has been some controversy relating to Grade Group 4, which includes Gleason scores $3+5=8$ and $5+3=8$, reporting that Gleason score 8 cases with pattern 5 has a worse prognosis from those with 4 $+4=8$ disease. ${ }^{102,103}$ One of these studies included patients from 1998 to 2012 and concluded that prostate cancer-specific mortality was higher when any percent of pattern 5 was present compared with Gleason score $4+4=8$ disease. ${ }^{102}$ No comparison was performed between the outcomes of Gleason score 3 $+5=8$ and $5+3=8$, and cases from before the 2005 International Society of Urological Pathology (ISUP) conference would have been graded significantly different from current practice. Another study extracted data from the Surveillance, Epidemiology, and End Results (SEER) database and concluded that prostate cancer-specific mortality was similar for Gleason score $4+4=8$ and $3+5=8$ prostate cancer, and the prognosis of patients with Gleason score 5 $+3=8$ was more similar to those with Gleason score 9 disease. ${ }^{103}$ Another recent study also showed that the prognosis is not different in Gleason score $3+5=8$ and $4+4=8$ cancers. ${ }^{104}$ While SEER provides a large database for retrospective prostate cancer research, it is not controlled for the consistency of reporting/ grading and the data are extracted from pathology reports in hospitals without central re-review of the slides, with most institutions lacking genitourinary pathology experts. One potential error is to grade the overall tumor as $5+3=8$ in an RP if there are separate nodules of $5+5=10$ and $3+3=6$. Similarly, a case may have been incorrectly issued a grade of $5+3=8$ on needle biopsy if there were separate cores with 5 $+5=10$ and $3+3=6$. In both these scenarios, the cases should be graded as $5+5=10$ so that it would be expected that their prognosis would be worse than Gleason score 8. In another study of Grade Group 4 tumors, 295 (27.1\%), 651 (59.8\%), and 143 (13.1\%) patients had pathologic Gleason scores $3+5,4+4$, and $5+3 .{ }^{105}$ The relatively high percentage of cases with $3+5$ and $5+3$ calls into question on how these tumors were graded. The study lacks a single pathologist amongst the authors, no details were provided on how the grades were assigned, and the grade was solely based on review of pathology reports. They reported patients with Gleason pattern $3+5$ were at lower risk of biochemical recurrence compared to those with $4+4$.

In a large multi-institutional study with genitourinary pathology experts from 2005 to 2014, out of 20845 RP specimens, there were $39(0.2 \%)$ cases with $3+5=8$ and $4(0.02 \%)$ with $5+3=8$ (unpublished data). ${ }^{92}$ Similarly, out of 16172 needle biopsy cases, there were only $44(0.3 \%)$ with $3+5=8$ and 6 $(0.04 \%)$ with $5+3=8$ (unpublished data). These data indicate that Gleason score $5+3=8$ cancer on needle biopsy or RP almost never occurs in clinical practice.

\section{Summary}

The grading of prostate cancer has evolved significantly since Gleason's original description in the 1960-70s. Based on ISUP consensus conferences in 2005 and 2015, many of the original Gleason pattern 3 morphologies have now been shifted to Gleason pattern 4 with a relatively better prognosis for current Gleason score 6 tumors. Even the basic definition of Gleason grading by adding the most prevalent and second most prevalent patterns to arrive at a score has changed on needle biopsy where the most prevalent pattern is added to the highest grade. Grading has also evolved with new rules for grading of prostate cancer variants and variant morphologies, as well as for needle biopsies and radical prostatectomies. A new simple, intuitive grading system has been accepted clinicians with 
better stratification of patients into distinct grade grades, which has implications for treatment. Grade Group 1/5 emphasizes the generally indolent behavior of Gleason score 6 prostate cancer to permit more rational and less emotional decision making for treatment, likely increasing the appropriate use of active surveillance in these men. Grade Group 2/5 can reassure patients of its very good prognosis with only rare metastases, as opposed to Gleason score 7, which is closer to Gleason score 10 than Gleason score 2, implying aggressive disease. Grade Group $3 / 5$ with a significantly worse prognosis than Grade 2 reinforces their distinction as opposed to Gleason score 7 , which combines Gleason scores $3+4$ and 4 +3. Grade Group 4/5 (Gleason score 8) is not considered the highest grade with a significantly better prognosis than Grade Group 5 (Gleason scores 9 and 10), as opposed to the typical combining of Gleason scores 8-10. Grade Group 5 obviates the need to distinguish between Gleason scores $4+5,5$ +4 , and $5+5$ just as Grade Group 1 makes irrelevant the distinction between Gleason scores 2-6.

\section{Disclosure/conflict of interest}

The authors declare no conflict interest.

\section{References}

1 Mellinger GT. Prognosis of prostatic carcinoma. Recent Results Cancer Res 1977;60:61-72.

2 Bailar JC III, Mellinger GT, Gleason DF. Survival rates of patients with prostatic cancer, tumor stage, and differentiation-preliminary report. Cancer Chemother Rep 1966;50:129-136.

3 Gleason DF. Histological grading and staging of prostatic carcinoma. In: Urologic Pathology: The Prostate. Tannenbaum M (ed). Lea and Feibiger: Philadelphia, PA, 1977, p 171.

4 Epstein JI. Gleason score 2-4 adenocarcinoma of the prostate on needle biopsy: a diagnosis that should not be made. Am J Surg Pathol 2000;24:477-478.

5 Fine SW, Epstein JI. A contemporary study correlating prostate needle biopsy and radical prostatectomy Gleason score. J Urol 2008;179:1335-1338.

6 Epstein JI, Allsbrook WC Jr, Amin MB et al. ISUP Grading Committee The 2005 international society of urological pathology (ISUP) consensus conference on Gleason grading of prostatic carcinoma. Am J Surg Pathol 2005;29:1228-1242.

7 Epstein JI, Egevad L, Amin MB, et al. The 2014 International Society of Urological Pathology (ISUP) consensus conference on Gleason grading of prostatic carcinoma: definition of grading patterns and proposal for a new grading system. Am J Surg Pathol 2016;40:244-252.

8 McNeal JE, Yemoto CE. Spread of adenocarcinoma within prostatic ducts and acini. morphologic and clinical correlations. Am J Surg Pathol 1996;20: 802-814

9 Ross HM, Kryvenko ON, Cowan JE, et al. Do adenocarcinomas of the prostate with Gleason score
(GS) $\leq 6$ have the potential to metastasize to lymph nodes? Am J Surg Pathol 2012;36:1346-1352.

10 Latour M, Amin MB, Billis A, et al. Grading of invasive cribriform carcinoma on prostate needle biopsy: an interobserver study among experts in genitourinary pathology. Am J Surg Pathol 2008;32: 1532-1539.

11 Iczkowski KA, Torkko KC, Kotnis GR, et al. Digital quantification of five high-grade prostate cancer patterns, including the cribriform pattern, and their association with adverse outcome. Am J Clin Pathol 2011;136:98-107.

12 Kweldam CF, Wildhagen MF, Steyerberg EW, et al. Cribriform growth is highly predictive for postoperative metastasis and disease-specific death in Gleason score 7 prostate cancer. Mod Pathol 2015;28:457-464.

13 Trudel D, Downes MR, Sykes J, et al. Prognostic impact of intraductal carcinoma and large cribriform carcinoma architecture after prostatectomy in a contemporary cohort. Eur J Cancer 2014;50: 1610-1616.

14 Baisden BL, Kahane H, Epstein JI. Perineural invasion, mucinous fibroplasia, and glomerulations: diagnostic features of limited cancer on prostate needle biopsy. Am J Surg Pathol 1999;23:918-924.

15 Lotan TL, Epstein JI. Gleason grading of prostatic adenocarcinoma with glomeruloid features on needle biopsy. Hum Pathol 2009;40:471-477.

16 Al-Hussain TO, Nagar MS, Epstein JI. Gleason pattern 5 is frequently underdiagnosed on prostate needlecore biopsy. Urology 2012;79:178-181.

17 Fajardo DA, Miyamoto H, Miller JS, et al. Identification of Gleason pattern 5 on prostatic needle core biopsy: frequency of underdiagnosis and relation to morphology. Am J Surg Pathol 2011;35: 1706-1711.

18 Epstein JI. Prostate cancer grading: a contemporary photomontage. Am J Surg Pathol 2016;40:137.

19 Nelson RS, Epstein JI. Prostatic carcinoma with abundant xanthomatous cytoplasm. Foamy gland carcinoma. Am J Surg Pathol 1996;20:419-426.

20 Zhao J, Epstein JI. High-grade foamy gland prostatic adenocarcinoma on biopsy or transurethral resection: a morphologic study of 55 cases. Am J Surg Pathol 2009;33:583-590.

21 Brinker DA, Potter SR, Epstein JI. Ductal adenocarcinoma of the prostate diagnosed on needle biopsy: correlation with clinical and radical prostatectomy findings and progression. Am J Surg Pathol 1999;23: 1471-1479.

22 Tavora F, Epstein JI. High-grade prostatic intraepithelial neoplasialike ductal adenocarcinoma of the prostate: a clinicopathologic study of 28 cases. Am J Surg Pathol 2008;32:1060-1067.

23 Ro JY, Grignon DJ, Ayala AG, et al. Mucinous adenocarcinoma of the prostate: histochemical and immunohistochemical studies. Hum Pathol 1990;21: 593-600.

24 Epstein JI, Lieberman PH. Mucinous adenocarcinoma of the prostate gland. Am J Surg Pathol 1985;9:299-308.

25 Osunkoya AO, Nielsen ME, Epstein JI. Prognosis of mucinous adenocarcinoma of the prostate treated by radical prostatectomy: a study of 47 cases. Am J Surg Pathol 2008;32:468-472.

26 Lane BR, Magi-Galluzzi C, Reuther AM, et al. Mucinous adenocarcinoma of the prostate does not confer poor prognosis. Urology 2006;68:825-830. 
27 Wang W, Epstein JI. Small cell carcinoma of the prostate. A morphologic and immunohistochemical study of 95 cases. Am J Surg Pathol 2008;32:65-71.

28 Levi AW, Epstein JI. Pseudohyperplastic prostatic adenocarcinoma on needle biopsy and simple prostatectomy. Am J Surg Pathol 2000;24:1039-1046.

29 Humphrey PA, Kaleem Z, Swanson PE, et al. Pseudohyperplastic prostatic adenocarcinoma. Am J Surg Pathol 1998;22:1239-1246.

30 Pan CC, Potter SR, Partin AW, et al. The prognostic significance of tertiary Gleason patterns of higher grade in radical prostatectomy specimens: a proposal to modify the Gleason grading system. Am J Surg Pathol 2000;24:563-569.

31 Hashine K, Yuasa A, Shinomori K, et al. Tertiary Gleason pattern 5 and oncological outcomes after radical prostatectomy. Jpn J Clin Oncol 2011;41: 571-576.

32 Hattab EM, Koch MO, Eble JN, et al. Tertiary Gleason pattern 5 is a powerful predictor of biochemical relapse in patients with Gleason score 7 prostatic adenocarcinoma. J Urol 2006;175:1695-1699.

33 Adam M, Hannah A, Budaus L, et al. Tertiary Gleason pattern in the prostatectomy specimen and its association with adverse outcome after radical prostatectomy. J Urol 2014;192:97-101.

34 Sim HG, Telesca D, Culp SH, et al. Tertiary Gleason pattern 5 in Gleason 7 prostate cancer predicts pathological stage and biochemical recurrence. J Urol 2008;179:1775-1779.

35 Turker P, Bas E, Bozkurt S, et al. Presence of high grade tertiary Gleason pattern upgrades the Gleason sum score and is inversely associated with biochemical recurrence-free survival. Urol Oncol 2013;31: 93-98.

36 Trock BJ, Guo CC, Gonzalgo ML, et al. Tertiary Gleason patterns and biochemical recurrence after prostatectomy: proposal for a modified Gleason scoring system. J Urol 2009;182:1364-1370.

37 van Oort IM, Schout BM, Kiemeney LA, et al. Does the tertiary Gleason pattern influence the PSA progression-free interval after retropubic radical prostatectomy for organ-confined prostate cancer? Eur Urol 2005;48:572-576.

38 Whittemore DE, Hick EJ, Carter MR, et al. Significance of tertiary Gleason pattern 5 in Gleason score 7 radical prostatectomy specimens. J Urol 2008;179: $516-522$.

39 Isbarn H, Ahyai SA, Chun FK, et al. Prevalence of a tertiary Gleason grade and its impact on adverse histopathologic parameters in a contemporary radical prostatectomy series. Eur Urol 2009;55:394-401.

40 Lucca I, Shariat SF, Briganti A, et al. Validation of tertiary Gleason pattern 5 in Gleason score 7 prostate cancer as an independent predictor of biochemical recurrence and development of a prognostic model. Urol Oncol 2015;33:e21-e26.

41 Baras AS, Nelson JB, Han M, et al. The effect of limited (tertiary) Gleason pattern 5 on the new prostate cancer grade groups. Hum Pathol 2017;63:27-32.

42 Choy B, Pearce SM, Anderson BB, et al. Prognostic significance of percentage and architectural types of contemporary Gleason pattern 4 prostate cancer in radical prostatectomy. Am J Surg Pathol 2016;40: 1400-1406.

43 Epstein JI, Amin MB, Reuter VE, et al. Contemporary Gleason grading of prostatic carcinoma: an update with discussion on practical issues to implement the 2014 International Society of Urological Pathology (ISUP) consensus conference on Gleason grading of prostatic carcinoma. Am J Surg Pathol 2017;41:e1-e7.

44 Moch H, Humphrey PA, Ulbright TM, et al. WHO Classification of Tumours of the Urinary System and Male Genital Organs. International Agency for Research on Cancer: Lyon, France, 2016.

45 Morash C, Tey R, Agbassi C, et al. Active surveillance for the management of localized prostate cancer: guideline recommendations. Can Urol Assoc J 2015;9: 171-178.

46 Chen RC, Rumble RB, Loblaw DA, et al. Active surveillance for the management of localized prostate cancer (Cancer Care Ontario Guideline): American Society of Clinical Oncology Clinical Practice Guideline Endorsement. J Clin Oncol 2016;34:2182-2190.

47 Zumsteg ZS, Spratt DE, Pei I, et al. A new risk classification system for therapeutic decision making with intermediate-risk prostate cancer patients undergoing dose-escalated external-beam radiation therapy. Eur Urol 2013;64:895-902.

48 Sadimin ET, Khani F, Diolombi M, et al. Interobserver reproducibility of percent Gleason pattern 4 in prostatic adenocarcinoma on prostate biopsies. Am J Surg Pathol 2016;40:1686-1692.

49 Egevad L, Delahunt B, Samaratunga H, et al. Utility of reporting the percentage of high-grade prostate cancer. Eur Urol 2016;69:599-600.

50 Deng FM, Donin NM, Pe Benito R, et al. Size-adjusted quantitative Gleason score as a predictor of biochemical recurrence after radical prostatectomy. Eur Urol 2016;70:248-253.

51 Sauter G, Steurer S, Clauditz TS, et al. Clinical utility of quantitative Gleason grading in prostate biopsies and prostatectomy specimens. Eur Urol 2016;69: 592-598

52 Cole AI, Morgan TM, Spratt DE, et al. Prognostic value of percent Gleason grade 4 at prostate biopsy in predicting prostatectomy pathology and recurrence. J Urol 2016;196:405-411.

53 Flood TA, Schieda N, Keefe DT, et al. Utility of Gleason pattern 4 morphologies detected on transrectal ultrasound (TRUS)-guided biopsies for prediction of upgrading or upstaging in Gleason score $3+4=7$ prostate cancer. Virchows Arch 2016;469:313-319.

54 Kuroiwa K, Uchino H, Yokomizo A, et al. Impact of reporting rules of biopsy Gleason score for prostate cancer. J Clin Pathol 2009;62:260-263.

55 Kunz GM Jr, Epstein JI. Should each core with prostate cancer be assigned a separate Gleason score? Hum Pathol 2003;34:911-914.

56 Park HK, Choe G, Byun SS, et al. Evaluation of concordance of Gleason score between prostatectomy and biopsies that show more than two different Gleason scores in positive cores. Urology 2006;67:110-114.

57 Poulos CK, Daggy JK, Cheng L. Preoperative prediction of Gleason grade in radical prostatectomy specimens: the influence of different Gleason grades from multiple positive biopsy sites. Mod Pathol 2005;18: 228-234.

58 Kunju LP, Daignault S, Wei JT, et al. Multiple prostate cancer cores with different Gleason grades submitted in the same specimen container without specific site designation: Should each core be assigned an individual Gleason score? Hum Pathol 2009;40: 558-564. 
59 Tolonen TT, Kujala PM, Tammela TL, et al. Overall and worst Gleason scores are equally good predictors of prostate cancer progression. BMC Urol 2011;11: 2490-11-21.

60 Berney DM, Beltran L, Fisher G, et al. Validation of a contemporary prostate cancer grading system using prostate cancer death as outcome. Br J Cancer 2016;114:1078-1083.

61 Eifler JB, Feng Z, Lin BM, et al. An updated prostate cancer staging nomogram (Partin tables) based on cases from 2006 to 2011. BJU Int 2013;111:22-29.

62 Ohori M, Kattan MW, Koh H, et al. Predicting the presence and side of extracapsular extension: a nomogram for staging prostate cancer. J Urol 2004;171:1844-1849.

63 Hall GS, Kramer CE, Epstein JI. Evaluation of radical prostatectomy specimens. A comparative analysis of sampling methods. Am J Surg Pathol 1992;16: 315-324.

64 Sehdev AE, Pan CC, Epstein JI. Comparative analysis of sampling methods for grossing radical prostatectomy specimens performed for nonpalpable (stage T1c) prostatic adenocarcinoma. Hum Pathol 2001;32: 494-499.

65 Cohen MB, Soloway MS, Murphy WM. Sampling of radical prostatectomy specimens. How much is adequate? Am J Clin Pathol 1994;101:250-252.

66 Chabner BA, Smith M. Call it cancer. Oncologist 2012;17:149-150.

67 Kweldam CF, Wildhagen MF, Bangma $\mathrm{CH}$, et al. Disease-specific death and metastasis do not occur in patients with Gleason score $</=6$ at radical prostatectomy. BJU Int 2015;116:230-235.

68 Hernandez DJ, Nielsen ME, Han M, et al. Natural history of pathologically organ-confined (pT2), Gleason score 6 or less, prostate cancer after radical prostatectomy. Urology 2008;72:172-176.

69 Miyamoto H, Hernandez DJ, Epstein JI. A pathological reassessment of organ-confined, Gleason score 6 prostatic adenocarcinomas that progress after radical prostatectomy. Hum Pathol 2009;40:1693-1698.

70 Esserman LJ, Thompson IM, Reid B, et al. Addressing overdiagnosis and overtreatment in cancer: a prescription for change. Lancet Oncol 2014;15: e234-e242.

71 Kooby DA, Antonescu CR, Brennan MF, et al. Atypical lipomatous tumor/well-differentiated liposarcoma of the extremity and trunk wall: importance of histological subtype with treatment recommendations. Ann Surg Oncol 2004;11:78-84.

72 Epstein JI, Amin MB, Reuter VR, et al. The world health Organization/International Society of Urological Pathology consensus classification of urothelial (transitional cell) neoplasms of the urinary bladder. bladder consensus conference committee. Am J Surg Pathol 1998;22:1435-1448.

73 Nikiforov YE, Seethala RR, Tallini G, et al. Nomenclature revision for encapsulated follicular variant of papillary thyroid carcinoma: a paradigm shift to reduce overtreatment of indolent tumors. JAMA Oncol 2016;2:1023-1029.

74 Berman DM, Epstein JI. When is prostate cancer really cancer? Urol Clin N Am 2014;41:339-346.

75 Netto GJ, Cheng L. Emerging critical role of molecular testing in diagnostic genitourinary pathology. Arch Pathol Lab Med 2012;136:372-390.
76 Epstein JI, Feng Z, Trock BJ, et al. Upgrading and downgrading of prostate cancer from biopsy to radical prostatectomy: incidence and predictive factors using the modified Gleason grading system and factoring in tertiary grades. Eur Urol 2012;61:1019-1024.

77 Loeb S, Curnyn C, Sedlander E. Perspectives of prostate cancer patients on Gleason scores and the new grade groups: initial qualitative study. Eur Urol 2016;70:1083-1085.

78 Resnick MJ, Koyama T, Fan KH, et al. Long-term functional outcomes after treatment for localized prostate cancer. N Engl J Med 2013;368:436-445.

79 Wilt TJ, Brawer MK, Jones KM et al. Prostate Cancer Intervention versus Observation Trial (PIVOT) Study Group Radical prostatectomy versus observation for localized prostate cancer. N Engl J Med 2012;367: 203-213.

80 Bill-Axelson A, Holmberg L, Garmo H, et al. Radical prostatectomy or watchful waiting in early prostate cancer. N Engl J Med 2014;370:932-942.

81 D'Amico AV, Whittington R, Malkowicz SB, et al. Biochemical outcome after radical prostatectomy, external beam radiation therapy, or interstitial radiation therapy for clinically localized prostate cancer. JAMA 1998;280:969-974.

82 Chan TY, Partin AW, Walsh PC, et al. Prognostic significance of Gleason score $3+4$ versus Gleason score $4+3$ tumor at radical prostatectomy. Urology 2000;56:823-827.

83 Makarov DV, Sanderson H, Partin AW, et al. Gleason score 7 prostate cancer on needle biopsy: Is the prognostic difference in Gleason scores $4+3$ and $3+4$ independent of the number of involved cores? J Urol 2002;167:2440-2442.

84 Amin A, Partin A, Epstein JI. Gleason score 7 prostate cancer on needle biopsy: relation of primary pattern 3 or 4 to pathological stage and progression after radical prostatectomy. J Urol 2011;186:1286-1290.

85 Pierorazio PM, Walsh PC, Partin AW, et al. Prognostic Gleason grade grouping: data based on the modified Gleason scoring system. BJU Int 2013;111:753-760.

86 Sabolch A, Feng FY, Daignault-Newton S, et al. Gleason pattern 5 is the greatest risk factor for clinical failure and death from prostate cancer after doseescalated radiation therapy and hormonal ablation. Int J Radiat Oncol Biol Phys 2011;81:e351-e360.

87 Stenmark MH, Blas K, Halverson S, et al. Continued benefit to androgen deprivation therapy for prostate cancer patients treated with dose-escalated radiation therapy across multiple definitions of high-risk disease. Int J Radiat Oncol Biol Phys 2011;81: e335-e344.

88 Stock RG, Cesaretti JA, Stone NN. Disease-specific survival following the brachytherapy management of prostate cancer. Int J Radiat Oncol Biol Phys 2006;64: 810-816.

89 Sylvester JE, Grimm PD, Wong J, et al. Fifteen-year biochemical relapse-free survival, cause-specific survival, and overall survival following I(125) prostate brachytherapy in clinically localized prostate cancer: Seattle experience. Int J Radiat Oncol Biol Phys 2011;81:376-381.

90 Stone NN, Stone MM, Rosenstein BS, et al. Influence of pretreatment and treatment factors on intermediate to long-term outcome after prostate brachytherapy. J Urol 2011;185:495-500. 
91 Djaladat $\mathrm{H}$, Amini $\mathrm{E}, \mathrm{Xu} \mathrm{W}$, et al. Oncological outcomes after radical prostatectomy for high-risk prostate cancer based on new Gleason grouping system: A validation study from university of southern california with 3,755 cases. Prostate 2017;77: 743-748.

92 Epstein JI, Zelefsky MJ, Sjoberg DD, et al. A contemporary prostate cancer grading system: a validated alternative to the Gleason score. Eur Urol 2016;69:428-435.

93 Mathieu R, Moschini M, Beyer B, et al. Prognostic value of the new grade groups in prostate cancer: a multi-institutional European validation study. Prostate Cancer Prostatic Dis 2017;20:197-202.

94 Thurtle D, Hsu RC, Chetan M, et al. Incorporating multiparametric MRI staging and the new histological grade group system improves risk-stratified detection of bone metastasis in prostate cancer. Br J Cancer 2016;115:1285-1288.

95 Yeong J, Sultana R, Teo J, et al. Gleason grade grouping of prostate cancer is of prognostic value in Asian men. J Clin Pathol 2017;70:745-753.

96 Pompe RS, Davis-Bondarenko $\mathrm{H}$, Zaffuto E, et al. Population-based validation of the 2014 ISUP Gleason grade groups in patients treated with radical prostatectomy, brachytherapy, external beam radiation, or no local treatment. Prostate 2017;77:686-693.

97 Leapman MS, Cowan JE, Simko J, et al. Application of a prognostic Gleason grade grouping system to assess distant prostate cancer outcomes. Eur Urol 2017;71: 750-759.
98 He J, Albertsen PC, Moore D, Rotter D, et al. Validation of a contemporary five-tiered Gleason grade grouping using population-based data. Eur Urol 2017;71:760-763.

99 Spratt DE, Jackson WC, Abugharib A, et al. Independent validation of the prognostic capacity of the ISUP prostate cancer grade grouping system for radiation treated patients with long-term follow-up. Prostate Cancer Prostatic Dis 2016;19:292-297.

100 Samaratunga H, Delahunt B, Gianduzzo T, et al. The prognostic significance of the 2014 International Society of Urological Pathology (ISUP) grading system for prostate cancer. Pathology 2015;47:515-519.

101 Loeb S, Folkvaljon Y, Robinson D, et al. Evaluation of the 2015 Gleason grade groups in a nationwide population-based cohort. Eur Urol 2016;69: 1135-1141.

102 Huynh MA, Chen MH, Wu J, et al. Gleason score 3+5 or $5+3$ versus $4+4$ prostate cancer: the risk of death. Eur Urol 2016;69:976-979.

103 Mahal BA, Muralidhar V, Chen YW, et al. Gleason score $5+3=8$ prostate cancer: much more like Gleason score 9? BJU Int 2016;118:95-101.

104 Harding-Jackson N, Kryvenko ON, Whittington EE, et al. Outcome of Gleason $3+5=8$ prostate cancer diagnosed on needle biopsy: prognostic comparison with Gleason 4+4=8. J Urol 2016;196:1076-1081.

105 Gandaglia G, Karnes RJ, Sivaraman A, et al. Are all grade group 4 prostate cancers created equal? implications for the applicability of the novel grade grouping. Urol Oncol 2017;35:e7-e14. 